\title{
Real-Time Kinematic Positioning with GPS and GLONASS
}

\author{
Patrick Henkel \\ Technische Universität München \\ Electrical and Computer Engineering \\ 80333 Munich, Germany \\ Email: patrick.henkel@tum.de
}

\author{
Ulrich Mittmann and Michele Iafrancesco \\ Advanced Navigation Solutions - ANavS GmbH \\ Precise Position and Attitude Determination Systems \\ 80798 Munich, Germany \\ Email: \{ulrich.mittmann, michele.iafrancesco\}@anavs.de
}

\begin{abstract}
Recently, low-cost dual constellation receivers with simultaneous tracking of GPS and GLONASS satellites and raw carrier phase output have become available. This enables a faster and more reliable ambiguity fixing especially in areas with limited satellite visibility, e.g. street canyons.

In this paper, we present a combined GPS/ GLONASS RTK positioning with code multipath estimation for low-cost receivers. We use double difference measurements and take the integer property of both the GPS and GLONASS ambiguities into account. For GLONASS, this requires a reparametrization of the double difference ambiguities and a subsequent parameter mapping.

The re-parameterized measurement models are used for ambiguity fixing with real measurements from two low-cost dual constellation GNSS receivers. We obtain residuals of a few centimeters for both GPS and GLONASS fixed carrier phases.
\end{abstract}

\section{INTRODUCTION}

Low-cost dual-constellation GNSS receivers with carrier phase measurement output have been developed recently. The increased number of simultaneously available satellites enables a faster and more reliable ambiguity fixing.

GLONASS uses a Frequency Division Multiple Access (FDMA) scheme, which results in a different carrier wavelength for every satellite. Consequently, the double difference combination of carrier phase integer ambiguities is no longer integer-valued. However, the double difference ambiguity combination can be represented as the linear combination of a double difference integer ambiguity and a single difference integer ambiguity. This representation has two consequences: The first one is a rank-defect due to the additional single difference ambiguity. The second consequence addresses the wavelength of the single difference ambiguity, which is in the order of only $65 \mu \mathrm{m}$. This small wavelength prevents any reliable fixing. Therefore, a parameter transformation is needed, which overcomes the rank-defect and still preserves the integer property.

We derive the transformation in section III. Section IV briefly describes the RTK positioning with combined GPS and GLONASS measurements. We estimate a code multipath parameter for every GPS and GLONASS satellite to exploit the time-correlation of multipath and to prevent a mapping of multipath to other state parameters. Finally, we present some measurement results in Section $\mathrm{V}$ and conclude this paper in Section VI.

\section{Measurement Model}

The undifferenced carrier phase measurement for the $k$-th GLONASS satellite of the $r$-th receiver is modeled as

$$
\begin{aligned}
\lambda_{k} \varphi_{r}^{k}= & \left\|\vec{x}_{r}-\vec{x}^{k}\right\|+c\left(\delta \tau_{r}-\delta \tau^{k}\right)-I_{r}^{k}+T_{r}^{k} \\
& +\lambda_{k} N_{r}^{k}+\tilde{\beta}_{r}\left(n_{k}\right)+\beta^{k}+\varepsilon_{r}^{k}
\end{aligned}
$$

with the wavelength $\lambda_{k}$, the carrier phase measurement $\varphi_{r}^{k}$ in units of cycles, the receiver position $\vec{x}_{r}$, the satellite position $\vec{x}^{k}$, the speed of light $c$ in vacuum, the receiver clock offset $\delta \tau_{r}$, the satellite clock offset $\delta \tau^{k}$, the ionospheric delay $I_{r}^{k}$, the tropospheric delay $T_{r}^{k}$, the integer ambiguity $N_{r}^{k}$, the receiver phase bias $\tilde{\beta}_{r}\left(n_{k}\right)$ being a linear function of the GLONASS channel number $n_{k}$, the satellite phase bias $\beta^{k}$ and the phase noise $\varepsilon_{r}^{k}$.

The receiver phase biases are generated by the receiver's digital signal processing chain (see Sleewaegen et al. [1][2]) and can be accurately modeled as the sum of a satelliteindependent bias $\beta_{0, r}$ and a bias $n_{k} \beta_{r}$ that is proportional to the GLONASS channel number (see Wanninger [3]):

$$
\tilde{\beta}_{r}\left(n_{k}\right)=\beta_{0, r}+n_{k} \beta_{r}
$$

The GPS carrier phase measurements are modeled similarly and we refer to our earlier publication [4] for more details.

The single difference (SD) between the measurements of receivers $r$ and $u$ eliminates the satellite clock offset and phase bias, i.e.

$$
\begin{aligned}
\lambda_{k} \varphi_{u r}^{k}= & \vec{e}_{u}^{k}\left(\vec{x}_{u}-\vec{x}^{k}\right)-\vec{e}_{r}^{k}\left(\vec{x}_{r}-\vec{x}^{k}\right) \\
& +c \delta \tau_{u r}-I_{u r}^{k}+T_{u r}^{k}+\lambda_{k} N_{u r}^{k}+\beta_{u r}+\varepsilon_{u r}^{k} \\
= & \vec{e}_{u}^{k} \vec{x}_{u r}+c_{u r}^{k}+c \delta \tau_{u r}-I_{u r}^{k}+T_{u r}^{k} \\
& +\lambda_{k} N_{u r}^{k}+\beta_{0, u r}+n_{k} \beta_{u r}+\varepsilon_{u r}^{k},
\end{aligned}
$$

with $c_{u r}^{k}$ being the synchronization correction between receivers $u$ and $r$ as described in [5][6][7].

The double difference (DD) further eliminates the differential receiver clock offset and phase bias, i.e.

$$
\begin{aligned}
\lambda_{k} \varphi_{u r}^{k}-\lambda_{l} \varphi_{u r}^{l}= & \vec{e}_{u}^{k l} \vec{x}_{u r}+c_{u r}^{k l}-I_{u r}^{k l}+T_{u r}^{k l} \\
& +\lambda_{k} N_{u r}^{k}-\lambda_{l} N_{u r}^{l} \\
& +\left(n_{k}-n_{l}\right) \beta_{u r}+\varepsilon_{u r}^{k l}
\end{aligned}
$$


We subtract all available a priori information including the synchronization correction, and the differential ionospheric and tropospheric delay estimates $\hat{I}_{u r}^{k l}$ and $\hat{T}_{u r}^{k l}$, i.e.

$$
\begin{aligned}
\lambda_{k} \tilde{\varphi}_{u r}^{k}-\lambda_{l} \tilde{\varphi}_{u r}^{l}= & \lambda_{k} \varphi_{u r}^{k}-\lambda_{l} \varphi_{u r}^{l} \\
& -c_{u r}^{k l}+\hat{I}_{u r}^{k l}-\hat{T}_{u r}^{k l} \\
= & \vec{e}_{u}^{k l} \vec{x}_{u r}-\Delta I_{u r}^{k l}+\Delta T_{u r}^{k l} \\
& +\lambda_{k} N_{u r}^{k}-\lambda_{l} N_{u r}^{l} \\
& +\left(n_{k}-n_{l}\right) \beta_{u r}+\varepsilon_{u r}^{k l},
\end{aligned}
$$

which leaves the relative position $\vec{x}_{u r}$, the residual atmospheric delays, the integer ambiguities $N_{u r}^{k}$ and $N_{u r}^{l}$, and the differential receiver phase bias $\beta_{u r}$ as unknowns.

The double difference ambiguity term can be rewritten as

$$
\lambda_{k} N_{u r}^{k}-\lambda_{l} N_{u r}^{l}=\lambda_{k} N_{u r}^{k l}+\left(\lambda_{k}-\lambda_{l}\right) N_{u r}^{l},
$$

with $N_{u r}^{k l}$ being the DD integer ambiguity and $N_{u r}^{l}$ being the SD integer ambiguity.

The differences of wavelengths can be further developed with $f_{k}=f_{0}+n_{k} \Delta f=\left(n_{0}+n_{k}\right) \Delta f$ as

$$
\begin{aligned}
\lambda_{k}-\lambda_{l} & =\frac{c}{f_{0}+n_{k} \Delta f}-\frac{c}{f_{0}+n_{l} \Delta f} \\
& =c \cdot\left(n_{l}-n_{k}\right) \frac{\Delta f}{f_{k} \cdot f_{l}}=\lambda_{k} \cdot \frac{n_{l}-n_{k}}{n_{0}+n_{l}},
\end{aligned}
$$

which is approximately $65 \mu \mathrm{m}$ for $n_{0}=f_{0} / \Delta f=2848$ and $n_{l}-n_{k}=1$.

Eq. (6) can be written in matrix-vector notation as

$$
\begin{aligned}
& {\left[\begin{array}{c}
\lambda_{1} N_{u r}^{1}-\lambda_{l} N_{u r}^{l} \\
\lambda_{2} N_{u r}^{2}-\lambda_{l} N_{u r}^{l} \\
\vdots \\
\lambda_{K} N_{u r}^{K}-\lambda_{l} N_{u r}^{l}
\end{array}\right]} \\
& =\underbrace{\left[\begin{array}{cccc|c}
\lambda_{1} & & & & \lambda_{1}-\lambda_{l} \\
& \lambda_{2} & & & \lambda_{2}-\lambda_{l} \\
& & \ddots & & \vdots \\
& & & \lambda_{K} & \lambda_{K}-\lambda_{l}
\end{array}\right]}_{\Lambda} \cdot\left[\begin{array}{c}
N_{u r}^{1 l} \\
N_{u r}^{2 l} \\
\vdots \\
N_{u r}^{K l} \\
\hline N_{u r}^{l}
\end{array}\right] .
\end{aligned}
$$

\section{Parameter Transformation}

The ambiguity coefficient matrix $\Lambda$ has $K+1$ columns but only $K$ rows, which results in a rank-deficient system of equations. Therefore, we map one DD ambiguity (here: the first one $N_{u r}^{1 l}$ ) to the SD ambiguity $N_{u r}^{l}$ and introduce $\tilde{N}_{u r}^{l}$ as

$$
\begin{aligned}
\tilde{N}_{u r}^{l} & :=N_{u r}^{l}+\frac{\lambda_{1}}{\lambda_{1}-\lambda_{l}} N_{u r}^{1 l} \\
& =N_{u r}^{l}+\frac{n_{0}+n_{l}}{n_{l}-n_{1}} N_{u r}^{1 l} .
\end{aligned}
$$

The other DD ambiguities have to adjusted respectively, i.e.

$$
\tilde{N}_{u r}^{k l}=N_{u r}^{k l}-\frac{n_{l}-n_{k}}{n_{l}-n_{1}} \cdot N_{u r}^{1 l} .
$$

Note that both $\tilde{N}_{u r}^{l}$ and $\tilde{N}_{u r}^{k l}$ are integer valued for any $N_{u r}^{1 l}$ only if $n_{l}-n_{1}$ is 1 .
Multiplying both the SD and DD ambiguities by $n_{l}-n_{1}$ ensures that the ambiguities are always integer valued, i.e.

$$
\begin{aligned}
\bar{N}_{u r}^{l} & =\left(n_{l}-n_{1}\right) \cdot \tilde{N}_{u r}^{l} \\
& =\left(n_{l}-n_{1}\right) \cdot N_{u r}^{l}+\left(n_{0}+n_{l}\right) \cdot N_{u r}^{1 l} \in \mathbb{Z} \\
\bar{N}_{u r}^{k l} & =\left(n_{l}-n_{1}\right) \cdot \tilde{N}_{u r}^{k l} \\
& =\left(n_{l}-n_{1}\right) \cdot N_{u r}^{k l}-\left(n_{l}-n_{k}\right) \cdot N_{u r}^{1 l} \in \mathbb{Z} .
\end{aligned}
$$

We rewrite Eq. (8) in terms of $\bar{N}_{u r}^{l}$ and $\bar{N}_{u r}^{k l}$ to obtain the following full-rank ambiguity transformation:

$$
\begin{aligned}
& {\left[\begin{array}{c}
\lambda_{1} N_{u r}^{1}-\lambda_{l} N_{u r}^{l} \\
\lambda_{2} N_{u r}^{2}-\lambda_{l} N_{u r}^{l} \\
\vdots \\
\lambda_{K} N_{u r}^{K}-\lambda_{l} N_{u r}^{l}
\end{array}\right]} \\
& =\left[\begin{array}{ccc|c}
0 & \cdots & 0 & \frac{\lambda_{1}-\lambda_{l}}{n_{l}-n_{1}} \\
\frac{\lambda_{2}}{n_{l}-n_{1}} & & & \frac{\lambda_{2}-\lambda_{l}}{n_{l}-n_{1}} \\
& \ddots & & \vdots \\
& & \frac{\lambda_{K}}{n_{l}-n_{1}} & \frac{\lambda_{K}-\lambda_{l}}{n_{l}-n_{1}}
\end{array}\right] \cdot\left[\begin{array}{c}
\bar{N}_{u r}^{2 l} \\
\vdots \\
\bar{N}_{u r}^{K l} \\
\hline N_{u r}^{l}
\end{array}\right] .
\end{aligned}
$$

We recommend to choose $n_{l}$ and $n_{1}$ such that $n_{l}-n_{1}=1$. In this case, the combined $K-2$ DD ambiguities $\bar{N}_{u r}^{k l}$ can be considered as integer-valued with wavelengths $\lambda_{k}, k \in$ $\{1, \ldots, K\}$, while the SD ambiguity $\bar{N}_{u r}^{l}$ is considered as real-valued due to the small differential wavelengths $\lambda_{k}-\lambda_{l}$.

The DD carrier phase measurements of Eq. (5) also include the unknown differential receiver phase bias $\beta_{u r}$, which can not be separated from the ambiguities. Therefore, we map the differential receiver phase bias $\beta_{u r}$ to the SD ambiguity $\bar{N}_{u r}^{l}$, i.e.

$$
\begin{aligned}
& \frac{\lambda_{k}-\lambda_{l}}{n_{l}-n_{1}} \cdot \bar{N}_{u r}^{l}+\left(n_{k}-n_{l}\right) \cdot \beta_{u r} \\
& =\frac{\lambda_{k}-\lambda_{l}}{n_{l}-n_{1}} \cdot\left(\bar{N}_{u r}^{l}+\frac{\left(n_{k}-n_{l}\right)\left(n_{l}-n_{1}\right)}{\lambda_{k}-\lambda_{l}} \cdot \beta_{u r}\right) \\
& =\frac{\lambda_{k}-\lambda_{l}}{n_{l}-n_{1}} \cdot(\bar{N}_{u r}^{l}-\underbrace{\frac{\left(n_{0}+n_{l}\right)\left(n_{l}-n_{1}\right)}{\lambda_{k}}}_{\gamma_{k}} \cdot \beta_{u r}),
\end{aligned}
$$

where the last identity was obtained with Eq. (7). The dependency of the joint SD ambiguity/ bias term on $\lambda_{k}$ is not desirable as only one SD parameter can be estimated. We replace $\lambda_{k}$ by $\lambda_{0}=c / f_{0}$ in $\gamma_{k}$ introduce the error term $\varepsilon$ :

$$
\frac{\lambda_{k}-\lambda_{l}}{n_{l}-n_{1}} \cdot\left(\bar{N}_{u r}^{l}-\gamma_{k} \beta_{u r}\right) \stackrel{!}{=} \frac{\lambda_{k}-\lambda_{l}}{n_{l}-n_{1}} \cdot\left(\bar{N}_{u r}^{l}-\gamma_{0} \beta_{u r}\right)+\varepsilon
$$

Solving for $\varepsilon$ yields

$$
\begin{aligned}
\varepsilon & =\frac{\lambda_{k}-\lambda_{l}}{n_{l}-n_{1}} \cdot\left(\gamma_{0}-\gamma_{k}\right) \cdot \beta_{u r} \\
& =\left(\lambda_{k}-\lambda_{l}\right) \cdot\left(n_{0}+n_{l}\right) \cdot\left(\frac{1}{\lambda_{0}}-\frac{1}{\lambda_{k}}\right) \cdot \beta_{u r} \\
& =\left(\lambda_{k}-\lambda_{l}\right) \cdot\left(n_{0}+n_{l}\right) \cdot\left(\frac{n_{k} \Delta f}{c}\right) \cdot \beta_{u r} .
\end{aligned}
$$


Replacing $\Delta f$ by $f_{l} /\left(n_{0}+n_{l}\right)$ further simplifies $\varepsilon$ to

$$
\varepsilon=\frac{\lambda_{k}-\lambda_{l}}{\lambda_{l}} \cdot n_{k} \cdot \beta_{u r}
$$

which is far below the millimeter-level for any relevant phase bias $\beta_{u r}$. Thus, we can neglect $\varepsilon$ and replace $\gamma_{k}$ by $\gamma_{0}$ in Eq. (13).

The DD carrier phase measurements of Eq. (5) can be written with Eq. (12) in matrix-vector notation as

$$
\begin{aligned}
& {\left[\begin{array}{c}
\lambda_{1} \tilde{\varphi}_{u r}^{1}-\lambda_{l} \tilde{\varphi}_{u r}^{l} \\
\lambda_{2} \tilde{\varphi}_{u r}^{2}-\lambda_{l} \tilde{\varphi}_{u r}^{l} \\
\vdots \\
\lambda_{K} \tilde{\varphi}_{u r}^{K}-\lambda_{l} \tilde{\varphi}_{u r}^{l}
\end{array}\right]} \\
& =\left[\begin{array}{cc}
\vec{e}_{u}^{1 l} & \frac{\lambda_{1}-\lambda_{l}}{n_{l}-n_{1}} \\
\vec{e}_{u}^{2 l} & \frac{\lambda_{2}-\lambda_{l}}{n_{l}-n_{1}} \\
\vdots & \\
\vec{e}_{u}^{K l} & \frac{\lambda_{K}-\lambda_{l}}{n_{l}-n_{1}}
\end{array}\right]\left[\begin{array}{c}
\vec{x}_{u r} \\
\bar{N}_{u r}^{l}-\gamma_{0} \beta_{u r}
\end{array}\right] \\
& +\left[\begin{array}{cc}
0 & 0 \\
\frac{\lambda_{2}}{n_{l}-n_{1}} & \\
& \\
& \\
&
\end{array}\right] \cdot\left[\begin{array}{c}
\lambda_{K} \\
\bar{N}_{u r}^{2 l} \\
\vdots \\
\bar{N}_{u r}^{K l}
\end{array}\right]+\left[\begin{array}{c}
\varepsilon_{u r}^{1 l} \\
\varepsilon_{u r}^{2 l} \\
\vdots \\
\varepsilon_{u r}^{K l}
\end{array}\right],
\end{aligned}
$$

where we have assumed that the residual atmospheric errors are negligible.

The DD pseudorange measurements of GLONASS are also affected by inter-frequency biases. We extend the model of [9], and map the DD code bias $b_{u r}^{k l}$ to the DD code multipath $\Delta \rho_{\mathrm{MP} \mathrm{P}_{r}}^{k l}$, i.e.

$$
\begin{aligned}
& {\left[\begin{array}{c}
\tilde{\rho}_{u r}^{1 l} \\
\vdots \\
\tilde{\rho}_{u r}^{K l}
\end{array}\right]=\left[\begin{array}{c}
\tilde{\rho}_{u r}^{1 l}-c_{u r}^{1 l} \\
\vdots \\
\tilde{\rho}_{u r}^{K l}-c_{u r}^{K l}
\end{array}\right]} \\
& =\left[\begin{array}{cccc}
\vec{e}_{u}^{1 l} & 1 & & \\
\vdots & & \ddots & \\
\vec{e}_{u}^{K l} & & & 1
\end{array}\right]\left[\begin{array}{c}
\vec{x}_{u r} \\
\Delta \rho_{\mathrm{MP}_{u r}^{1 l}}^{1 l}+b_{u r}^{1 l} \\
\vdots \\
\Delta \rho_{\mathrm{MP}}^{K l}+b_{u r}^{K l}
\end{array}\right]+\left[\begin{array}{c}
\eta_{u r}^{1 l} \\
\vdots \\
\eta_{u r}^{K l}
\end{array}\right]
\end{aligned}
$$

\section{RTK POSITIONING WITH GPS AND GLONASS}

In this section, the measurements of GPS and GLONASS are considered jointly. Therefore, we extend the notation by introducing the superscript labels GPS and GLO as constellation identifiers. The combined measurement vector is written as

$$
y=\left(\begin{array}{l}
y^{\mathrm{GPS}} \\
y^{\mathrm{GLO}}
\end{array}\right)
$$

with

$$
y^{\mathrm{GPS}}=\left(\begin{array}{c}
y_{\varphi}^{\mathrm{GPS}} \\
y_{\rho}^{\mathrm{GPS}}
\end{array}\right)=\left(\begin{array}{c}
\lambda \tilde{\varphi}_{u r}^{1 l, \mathrm{GPS}} \\
\vdots \\
\lambda \tilde{\varphi}_{u r}^{K l, \mathrm{GPS}} \\
\tilde{\rho}_{u r}^{1 l, \mathrm{GPS}} \\
\vdots \\
\tilde{\rho}_{u r}^{K l, \mathrm{GPS}}
\end{array}\right)
$$

and

$$
y^{\mathrm{GLO}}=\left(\begin{array}{c}
y_{\varphi}^{\mathrm{GLO}} \\
y_{\rho}^{\mathrm{GLO}}
\end{array}\right)=\left(\begin{array}{c}
\lambda_{1}^{\mathrm{GLO}} \tilde{\varphi}_{u r}^{1, \mathrm{GLO}}-\lambda_{l}^{\mathrm{GLO}} \tilde{\varphi}_{u r}^{l, \mathrm{GLO}} \\
\vdots \\
\frac{\lambda_{K}^{\mathrm{GLO}} \tilde{\varphi}_{u r}^{K, \mathrm{GLO}}-\lambda_{l}^{\mathrm{GLO}} \tilde{\varphi}_{u r}^{l, \mathrm{GLO}}}{\tilde{\rho}_{u r}^{1 l, \mathrm{GLO}}} \\
\vdots \\
\tilde{\rho}_{u r}^{\mathrm{KL}, \mathrm{GLO}}
\end{array}\right) .
$$

The unknown parameters are stacked in the state vector

$$
\begin{aligned}
& x=(\vec{x}_{u r}^{\mathrm{T}}, \underbrace{\Delta \rho_{\mathrm{MP}_{u r}^{1 l}}^{\mathrm{GPS}}, \ldots, \Delta \rho_{\mathrm{MP}_{u r}^{K l}}^{\mathrm{GPS}}}_{\in \mathbb{R}}, \\
& \underbrace{\bar{N}_{u r}^{l, \mathrm{GLO}}-\gamma_{0} \beta_{u r}^{\mathrm{GLO}}}_{\in \mathbb{R}}, \\
& \underbrace{\Delta \rho_{\mathrm{MP}_{u r}^{1 l}}^{\mathrm{GLO}}+b_{u r}^{1 l, \mathrm{GLO}}, \ldots, \Delta \rho_{\mathrm{MP}_{u r}^{K l}}^{\mathrm{GLO}}+b_{u r}^{K l, \mathrm{GLO}}}_{\in \mathbb{R}}, \\
& \underbrace{N_{u r}^{1 l, \mathrm{GPS}}, \ldots, N_{u r}^{K l, \mathrm{GPS}}, \bar{N}_{u r}^{2 l, \mathrm{GLO}}, \ldots \bar{N}_{u r}^{K l, \mathrm{GLO}}}_{\in \mathbb{Z}})^{\mathrm{T}}
\end{aligned}
$$

We use a standard Kalman filter [10] to determine the realvalued float solution $\hat{x}$ from $y$, and subsequently apply the LAMBDA method of Teunissen [8] with the extensions of Henkel and Sperl [9] for fixing of the DD GPS and GLONASS ambiguities.

Once the ambiguities are fixed, it is sufficient to consider the fixed phase measurements given by

$\check{y}=\left(y_{\varphi}^{\mathrm{GLO}}-\left(\begin{array}{ccc}0 & y_{\varphi}^{\mathrm{GPS}}-\lambda \check{N}_{u r}^{\mathrm{GPS}} \\ \frac{\lambda_{2}}{n_{l}-n_{1}} & \cdots & 0 \\ & \ddots & \\ & & \frac{\lambda_{K}}{n_{l}-n_{1}}\end{array}\right) \cdot\left(\begin{array}{c}\check{\bar{N}}_{u r}^{2 l, \mathrm{GLO}} \\ \vdots \\ \check{\bar{N}}_{u r}^{K l, \mathrm{GLO}}\end{array}\right)\right)$.

The fixed least-squares estimates of the baseline and SD ambiguity are obtained from the measurement model of Eq. (17) and given by

$$
\left[\begin{array}{c}
\check{\vec{x}}_{u r} \\
\check{\tilde{N}}_{u r}^{l, \mathrm{GLO}}-\gamma_{0} \check{\beta}_{u r}^{\mathrm{GLO}}
\end{array}\right]=\left(H^{\mathrm{T}} \Sigma^{-1} H\right)^{-1} H^{\mathrm{T}} \Sigma^{-1} \check{y},
$$

with

$$
H=\left(\begin{array}{cc}
H^{\mathrm{GPS}} & 0 \\
H^{\mathrm{GLO}} & a^{\mathrm{GLO}}
\end{array}\right)
$$


with the sub-matrices

$$
H^{\mathrm{GPS}}=H^{\mathrm{GLO}}=\left(\begin{array}{c}
\vec{e}_{u}^{1 l} \\
\vdots \\
\vec{e}_{u}^{K l}
\end{array}\right) \quad \text { and } \quad a^{\mathrm{GLO}}=\left(\begin{array}{c}
\frac{\lambda_{1}-\lambda_{l}}{n_{l}-n_{1}} \\
\vdots \\
\frac{\lambda_{K}-\lambda_{l}}{n_{l}-n_{1}}
\end{array}\right) \text {, }
$$

and the measurement covariance matrix $\Sigma$.

The accuracy of the least-squares estimation can be analyzed with the measurement residuals given by

$$
r_{\check{y}}=P_{H}^{\perp} \check{y} \quad \text { with } \quad P_{H}^{\perp}=1-H\left(H^{\mathrm{T}} \Sigma^{-1} H\right)^{-1} H^{\mathrm{T}} \Sigma^{-1} .
$$

\section{Measurement Results}

In this section, we analyze the performance of RTK positioning and attitude determination using two multi-sensor modules from ANavS and the measurements from a virtual reference station (VRS). Each multi-sensor module includes a low-cost LEA M8T multi-GNSS receiver from u-blox, a GNSS patch antenna, a low-cost inertial sensor from Invensense (MPU 9150) and a barometer from Bosch Sensortec. The multi-sensor modules were mounted on the roof of a car for joint RTK position and attitude determination as decribed in [7][9]. The VRS and the reference station network were operated by Axionet and the measurements were received with the NTRIP client. The length of the attitude baseline on the vehicle was $80 \mathrm{~cm}$ and the length of the RTK baseline between the vehicle and the VRS was $5 \mathrm{~km}$.

Fig. 1 and 2 show the fixed DD phase residuals of the attitude baseline for both GPS and GLONASS. The residuals of all satellites are drift-free with a magnitude of clearly less than 1 cycle, which indicates a correct ambiguity fixing and a positioning accuracy of $2 \mathrm{~cm}$. The residuals of GLONASS are almost unbiased whereas the GPS DD phase residuals show some biases of up to $2 \mathrm{~cm}$ over the considered time period of 13 minutes. Potential reasons for these small biases are phase multipath, wrong ambiguity fixings, the projection of GLONASS errors into GPS or unmodeled biases. We considered simultaneous measurements from multiple attitude baselines using receivers of the same type to get a better insight. We obtained different biases and believe that phase multipath is the primary source of these biases for low-cost receivers.

Fig. 3 and 4 show the fixed phase residuals of both GPS and GLONASS for the RTK baseline. The result is similar to the attitude baseline, i.e. the residuals of all satellites are again drift-free with a magnitude of clearly less than 1 cycle, which indicates a correct ambiguity fixing. The GPS residuals have again some small biases while the GLONASS residuals are almost unbiased. This can be explained to some extent by the real-valued scalar parameter $\bar{N}_{u r}^{l}-\gamma_{0} \beta_{u r}$ of GLONASS, which can absorb some biases. Obviously, the residual atmospheric errors are negligible for the considered baseline length of $5 \mathrm{~km}$ as the GLONASS residuals remain unbiased.

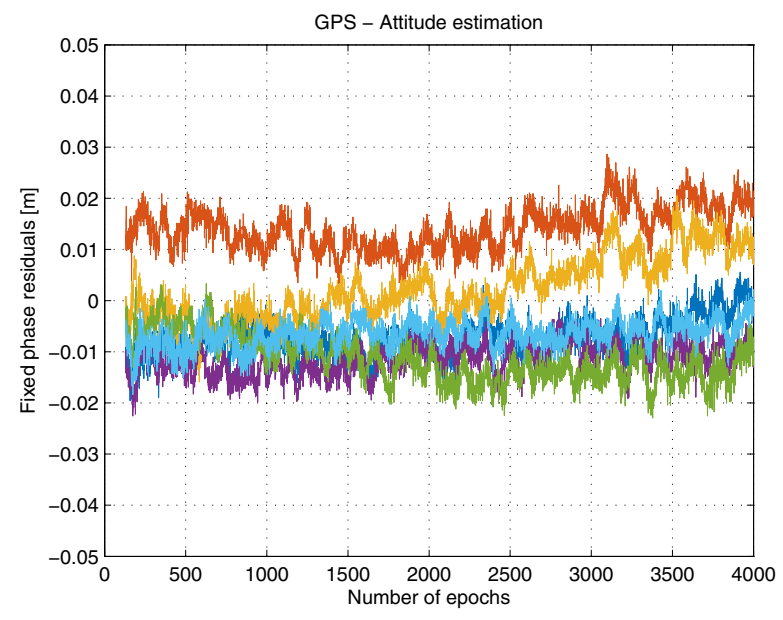

Fig. 1: Residuals of fixed GPS double difference carrier phase measurements for the short attitude baseline

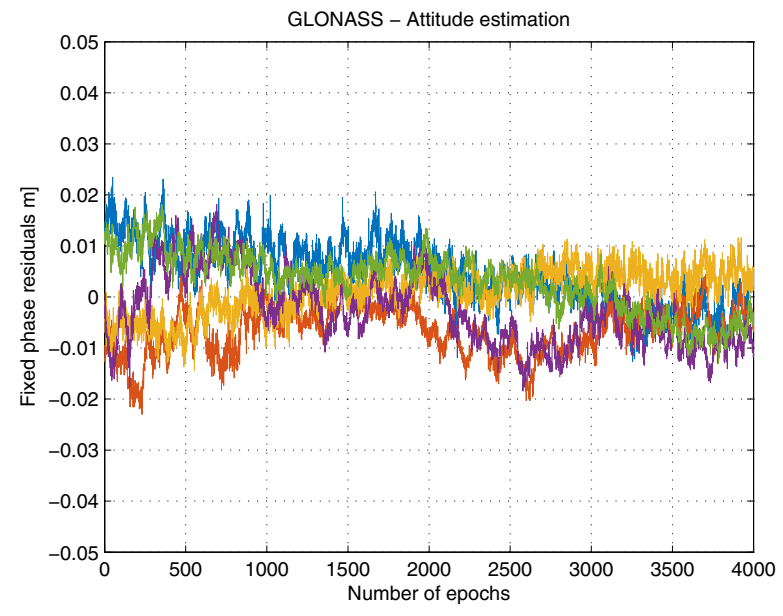

Fig. 2: Residuals of fixed GLONASS double difference carrier phase measurements for the short attitude baseline

\section{Conclusion}

In this paper, a method for RTK positioning with GPS and GLONASS measurements from low-cost GNSS receivers was described. The GLONASS double difference ambiguities are decomposed into an integer-valued double difference ambiguity for every satellite and a common single difference ambiguity. The common ambiguity is treated as real-valued as the wavelength is too small for ambiguity resolution. We also estimate the interfrequency phase and code biases and pseudorange multipath.

The GPS/ GLONASS dual constellation RTK was verified with real measurements from low-cost ANavS multi-sensor modules and a Virtual Reference Station from Axionet. The residuals of the fixed GPS and GLONASS carrier phase measurements were less than $2 \mathrm{cn}$ for most of the time, which indicates a correct ambiguity fixing and results in a millimeterto centimeter-level positioning accuracy. 


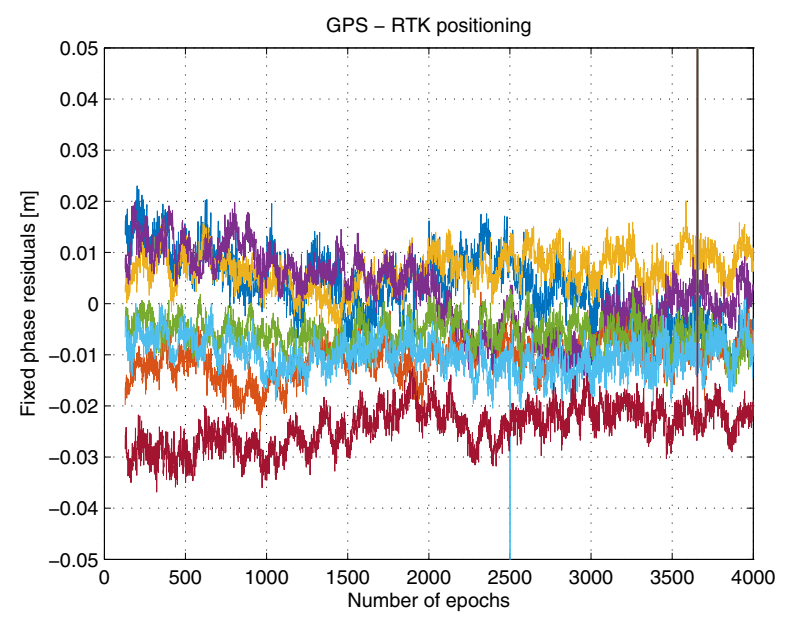

Fig. 3: Residuals of fixed GPS double difference carrier phase measurements for the RTK baseline with a baseline length of $5 \mathrm{~km}$.

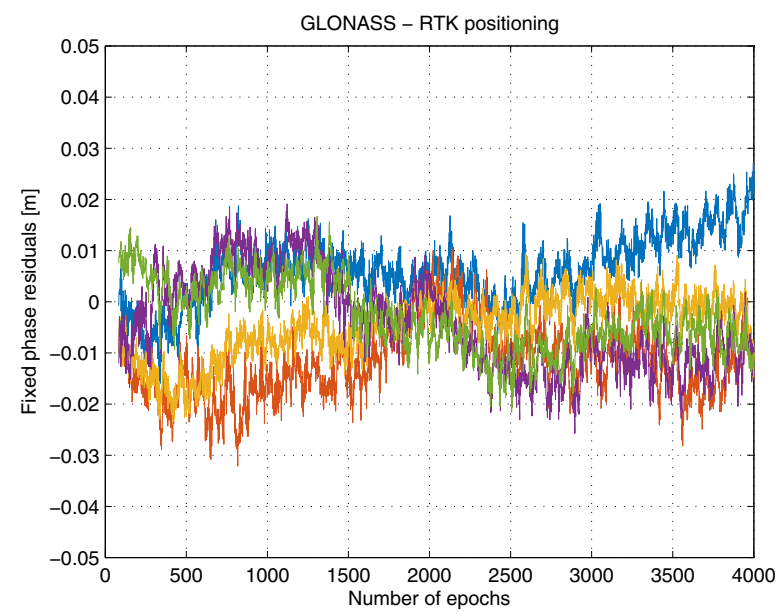

Fig. 4: Residuals of fixed GLONASS double difference carrier phase measurements for the RTK baseline with a baseline length of $5 \mathrm{~km}$.

\section{REFERENCES}

[1] J.-M. Sleewaegen, A. Simsky, W. de Wilde, F. Boon and T. Willems, Origin and Compensation of GLONASS Inter-frequency Carrier Phase Biases in GNSS Receivers, Proc. of 25-th Intern. Techn. Meeting of the Institute of Navigation (ION), Nashville, USA, pp. 2995 - 3001, Sep. 2012.

[2] J.-M. Sleewaegen, A. Simsky, W. de Wilde, F. Boon and T. Willems, Demystifying GLONASS Inter-Frequency Carrier Phase Biases, Inside GNSS, pp. 57 - 61, May 2012.

[3] L. Wanninger, Carrier-phase inter-frequency biases of GLONASS receivers, Journal of Geodesy, vol. 86, iss. 2, pp. 139 - 148, 2012.

[4] P. Henkel, M. Iafrancesco and A. Sperl, Precise Point Positioning with Multipath Estimation, Proc. of IEEE/ ION Position, Location and Navigation Symposium (PLANS), Savannah, GA, USA, DOI: 10.1109/PLANS.2016.7479694, pp. 144 - 149, Apr. 2016.

[5] P. Henkel and J. Kiam, Maximum A Posteriori Probability Estimation of Integer Ambiguities and Baseline, Proc. of 55-th IEEE Symp. ELMAR, pp. 353 - 356, Zadar, Croatia, Sep. 2013.

[6] Patrick S. Henkel and Michele Iafrancesco, Tightly coupled Position and Attitude Determination with two low-cost GNSS receivers, Proc. of 11- th Intern. IEEE Symp. on Wireless Communication Systems (ISWCS), Barcelona, Spain, pp. 895 - 900, Aug. 2014.

[7] P. Henkel, Tightly coupled precise point positioning and attitude determination, IEEE Transactions on Aerospace and Electronic Systems, vol. 51, iss. 4, pp. $3182-3197$, Oct. 2015.

[8] P. Teunissen, The least-squares ambiguity decorrelation adjustment: a method for fast GPS integer ambiguity estimation, Journal of Geodesy, vol. 70, pp. 65-82, 1995.

[9] P. Henkel and A. Sperl, Precise RTK Positioning with GPS/ INS Tight Coupling and Multipath Estimation, Proc. of the 2016 Intern. Techn. Meeting of the Institute of Navigation (ION), Monterey, USA, pp. 1015 $-1023,2016$.

[10] R. Brown and P. Hwang, Introduction to Random Signals and Applied Kalman Filtering with Matlab Exercises, 4th edition, 2012.

[11] S. Caranague, Low-cost GPS/GLONASS Precise Positioning Algorithm in Constrained Environment, $\mathrm{PhD}$ thesis, Universite de Toulouse, Feb. 2013. 